\title{
CARTESIAN CLOSED TOPOLOGICAL HULLS
}

\author{
H. HERRLICH AND L. D. NEL ${ }^{1}$
}

\begin{abstract}
It is shown in this paper that if a concrete category $\mathscr{A}$ admits embedding as a full finitely productive subcategory of a cartesian closed topological (CCT) category, then $\mathfrak{A}$ admits such embedding into a smallest CCT category, its CCT hull. This hull is characterized internally by means of density properties and externally by means of a universal property. The problem is posed of whether every topological category has a CCT hull.
\end{abstract}

Introduction. The category Top of topological spaces and continuous maps is not cartesian closed, hence inconvenient for many purposes in homotopy theory, topological algebra and functional analysis. Fortunately it can be fully embedded into some cartesian closed topological categories (briefly, CCT categories) such as the category Conv of convergence spaces (cf. D. C. Kent [21] and L. D. Nel [27]), the smaller category Lim of limit spaces (cf. H. J. Kowalsky [23], H. R. Fischer [14], A. Bastiani [2], C. H. Cook and H. R. Fischer [12], E. Binz and H. H. Keller [6], E. Binz [4], [5], A. Machado [24] and others) and the even smaller category Pstop of pseudotopological $\left(=L^{*}-\right)$ spaces (cf. G. Choquet [11], H. Poppe [29], A. Machado [24], L. D. Nel [27]). Recent work of P. Antoine [1] and A. Machado [24] has brought to light the existence of a smallest full CCT subcategory of Lim which contains Top. G. Bourdaud [7], [8], [9] studied smallest full CCT subcategories of Lim containing the categories of pretopological spaces and of uniformisable topological spaces respectively.

In this paper we will show that any concrete category $\mathfrak{A}$ with finite products which can be fully embedded in some CCT category such that the embedding is dense or preserves finite products, can be likewise embedded in a smallest CCT category, called the cartesian closed topological hull of $\mathfrak{A}$. This hull will be characterized internally by means of suitable density properties and externally by means of a universal property.

Preliminaries. All categories in this paper are supposed to be concrete, i.e., equipped with an underlying (faithful and amnestic ${ }^{2}$ ) functor into Set. A

Received by the editors April 27, 1976 and, in revised form, June 16, 1976.

AMS (MOS) subject classifications (1970). Primary 18D15, 18D99, 54A99; Secondary 18B15, $54 \mathrm{C} 35$.

Key words and phrases. Cartesian closed topological hull, dense functorial embedding, initial source preserving, power preserving, concrete category.

1 This research was aided by NRC grant A5297.

2 A faithful functor $U: \mathfrak{A} \rightarrow \mathfrak{X}$ is called amnestic if an $\mathfrak{A}$-isomorphism $f$ is an $\mathfrak{X}$-identity whenever $U f$ is an $\mathfrak{X}$-identity. 
functor will be called an embedding if it is full, faithful, injective on objects and preserves underlying sets. All subcategories are full and isomorphism-closed; thus typically their embedding functors are embeddings in the above sense. A category $\mathfrak{A}$ is called topological if it satisfies the following conditions:

$\mathfrak{A}$ is initially complete ${ }^{3}$;

$\mathfrak{A}$ has small fibres;

The fibre of a set with cardinality one consists of just one object.

A category $\mathfrak{A}$ is called cartesian closed provided:

$\mathfrak{U}$ has finite products and, for any $\mathfrak{U}$-object $A$ the functor $A \times-: \mathfrak{U} \rightarrow \mathfrak{A}$ has a right adjoint. It is known (H. Herrlich [17]) that a topological category is cartesian closed iff for any pair $(A, B)$ of $\mathfrak{X}$-objects the morphism set $\mathfrak{A}(A, B)$ can be equipped with the structure of an $\mathfrak{A}$-object (called a power and denoted by $\mathfrak{X}[A, B])$ such that:

The evaluation map $e: A \times \mathfrak{U}[A, B] \rightarrow B$ is an $\mathfrak{A}$-morphism;

for any $\mathfrak{A}$-morphism $f: A \times C \rightarrow B$ there exists a unique $\mathfrak{A}$-morphism $f^{*}: C \rightarrow \mathfrak{A}[A, B]$ with $e \circ\left(\operatorname{id}_{A} \times f^{*}\right)=f$.

If a category is said to have finite products then we will assume that these are preserved by the underlying functor (hence they are initial sources). A subcategory $\mathfrak{A}$ of $\mathfrak{B}$ is called dense (resp. codense) in $\mathfrak{B}$ provided that for any $\mathfrak{B}$-object $B$ there exists a final epi-sink $\left(f_{i}: A_{i} \rightarrow B\right)_{I}$ (resp. an initial source $\left.\left(f_{i}: B \rightarrow A_{i}\right)_{I}\right)$ with all $A_{i}$ belonging to $\mathfrak{A}$.

Readers desiring background information may find this for categories generally in H. Herrlich and G. E. Strecker [15]; for initial sources and final sinks in H. Herrlich [16]; for CCT categories in H. Herrlich [17], [18], L. D. Nel [27], [28] and O. Wyler [32], [33]. We recall two facts for convenient reference:

0. Proposition. (a) The coreflective hull of any finitely productive subcategory of a CCT category is a CCT category (see [27]).

(b) A topological category $\mathfrak{A}$ is a CCT category iff for any $\mathfrak{A}$-object $A$ the functor $A \times-: \mathfrak{A} \rightarrow \mathfrak{A}$ preserves final epi-sinks (see [17]).

Results. Henceforth let

$\mathfrak{D}$ be an arbitrary CCT category,

$\mathfrak{U}$ be a subcategory of $\mathfrak{D}$ closed under the formation of finite products in $\mathfrak{D}$,

$\mathfrak{C}$ be the coreflective hull of $\mathfrak{A}$ in $\mathfrak{D}$,

$\mathfrak{B}$ be the bireflective hull in $\mathbb{E}$ of the class of all $\mathbb{E}$-powers $\mathbb{E}\left[A, A^{\prime}\right]$ of $\mathfrak{A}$ obiects $A, A^{\prime}$.

Thus $\mathfrak{A} \subset \mathfrak{B} \subset \mathfrak{E} \subset \mathfrak{D}$. Further, let $\mathfrak{X}$ be an arbitrary subcategory of $\mathfrak{E}$ containing $\mathfrak{A}$.

1. Proposition. $\mathfrak{A}$ is dense in $\mathfrak{E}$.

\footnotetext{
${ }^{3} \mathrm{~A}$ source $\left(f_{i}: A \rightarrow A_{i}\right)_{I}$ in $\mathfrak{A}$ is called initial provided a function $g: B \rightarrow A$ is an $\mathfrak{A}$-morphism iff all compositions $f_{i} \circ g$ are $\mathfrak{A}$-morphisms. $\mathscr{A}$ is initially complete or is said to have initial sources

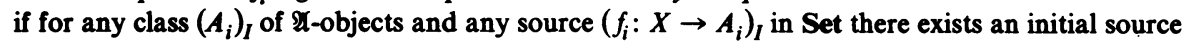
$\left(f_{i}: A \rightarrow A_{i}\right)_{I}$ in $\mathscr{A}$ with $U A=X$.
} 
2. Proposition. E is a CCT category.

Proof. Proposition O(a).

3. REMARK. In general, powers in $\mathbb{E}$ and in $\mathfrak{D}$ are formed differently. $\mathfrak{E}$ need not even be closed under the formation of $\mathfrak{D}$-powers $\mathfrak{D}\left[A, A^{\prime}\right]$ of $\mathfrak{U}$-objects. A counterexample is provided by $\mathfrak{U}=\mathfrak{E}=$ the category Conv $_{0}$ of symmetric convergence spaces and $\mathfrak{D}$ the category Grill of grill-determined nearness spaces (see H. L. Bentley et al. [3]).

4. Proposition. The following are equivalent:

(a) $\mathfrak{X}$ is a topological category,

(b) $\mathfrak{X}$ is a bireflective subcategory of $\mathfrak{E}$,

(c) $\mathfrak{X}$ is closed under formation of initial sources in $\mathbb{E}$.

Proof. Obviously (b) implies (a). The converse follows immediately from Proposition 1 and the result of $\mathbf{H}$. Müller [26] that any topological subcategory of a topological cateogry $\mathbb{E}$ is a bireflective subcategory of a coreflective subcategory of $\mathfrak{c}$. Equivalence of (b) and (c) is immediate.

5. Proposition. The following are equivalent:

(a) $¥$ is a CCT category.

(b) $\mathfrak{X}$ is bireflective in $\mathbb{C}$ and closed under the formation of powers in $\mathfrak{E}$.

Proof. By Proposition 4, (b) implies (a). For the converse it remains to show that $\mathfrak{X}\left[X, X^{\prime}\right]=\mathfrak{E}\left[X, X^{\prime}\right]$ for any pair $\left(X, X^{\prime}\right)$ of $\mathfrak{X}$-objects. Note [17] that both objects have the same underlying set $\mathbb{E}\left(X, X^{\prime}\right)$. So we need only show that the identity function on $\mathfrak{E}\left(X, X^{\prime}\right)$ is a morphism $\mathfrak{X}\left[X, X^{\prime}\right] \rightarrow \mathfrak{E}\left[X, X^{\prime}\right]$ as well as a morphism $\mathfrak{E}\left[X, X^{\prime}\right] \rightarrow \mathfrak{X}\left[X, X^{\prime}\right]$. The first morphism is obtained by taking the image of the evaluation $X \times \mathfrak{X}\left[X, X^{\prime}\right] \rightarrow X^{\prime}$ under the natural bijection $\mathfrak{E}\left(X \times \mathfrak{X}\left[X, X^{\prime}\right], X^{\prime}\right) \rightarrow \mathfrak{E}\left(\mathfrak{X}\left[X, X^{\prime}\right], \mathfrak{S}\left[X, X^{\prime}\right]\right)$. For the second observe that for any $\mathfrak{A}$-object $A$ we have the natural bijections $\mathbb{E}\left(A, \mathbb{\Im}\left[X, X^{\prime}\right]\right) \simeq \mathbb{E}\left(A \times X, X^{\prime}\right)$ $=\mathfrak{X}\left(A \times X, X^{\prime}\right) \simeq \mathfrak{X}\left(A, \mathfrak{X}\left[X, X^{\prime}\right]\right)$ since $\mathfrak{X}$ is closed under the formation of products in $\mathbb{E}$. This together with the fact that the sink $\left(f_{i}: A_{i} \rightarrow \mathbb{E}\left[X, X^{\prime}\right]\right)_{1}$, consisting of all morphisms with domain $A_{i}$ in $\mathfrak{A}$, is a final epi-sink in $\sqrt{5}$ provides the second morphism. The referee pointed out that (b) follows from (a) also by virtue of the main result in B. Day [13], which moreover implies that (a) and (b) are equivalent with

(c) $\mathfrak{X}$ is bireflective in $\mathbb{E}$ and the reflector preserves finite products.

6. Lemma. For any $\mathfrak{D}$-object $D$ the functor $\mathfrak{D}[D,-]: \mathfrak{D} \rightarrow \mathfrak{D}$ preserves initial sources and the contravariant functor $\mathfrak{D}[-, D]: \mathscr{D} \rightarrow \mathfrak{D}$ transforms final epi-sinks to initial sources.

Proof. Routine verification, using $0(b)$ for the second part.

7. Proposition. $\mathfrak{B}$ is closed under the formation of powers in $\mathfrak{E}$. Moreover $\mathfrak{夭}[C, B]$ belongs to $\mathfrak{B}$ for any $B$ in $\mathfrak{B}, C$ in $\mathfrak{夭}$. 
Proof. We have in $\mathbb{E}$ an initial source $\left(f_{i}: B \rightarrow \mathbb{E}\left[A_{i}, A_{i}^{\prime}\right]\right)_{I}$ and a final episink $\left(g_{j}: A_{j} \rightarrow C\right)_{J}$ with all $A_{i}, A_{i}^{\prime}, A_{j}$ in $\mathfrak{A}$. By Lemma 6,

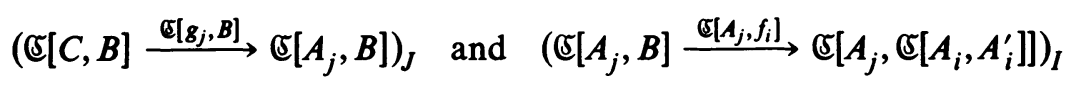

are initial sources for each $j$ in $J$. The $i$ th codomain is isomorphic to હ $\left[A_{j} \times A_{i}, A_{i}^{\prime}\right]$. By composing the above two sources we obtain an initial source of the form

$$
\left(\mathbb{E}[C, B] \stackrel{h_{i j}}{\longrightarrow} \mathbb{E}\left[A_{j} \times A_{i}, A_{i}^{\prime}\right]\right)_{I \times J}
$$

Since $\mathfrak{A}$ is closed under finite products, we conclude that $\mathbb{E}[C, B]$ belongs to $\mathfrak{B}$. By combining Propositions 4, 5 and 7 we obtain the following result.

8. THEOREM. $\mathfrak{B}$ is the smallest CCT subcategory of $\mathfrak{E}$ containing $\mathfrak{A}$.

By the above theorem, $\mathfrak{B}$ is uniquely determined as the smallest CCT subcategory of $\mathbb{E}$ containing $\mathfrak{A}$. Surprisingly enough, $\mathfrak{B}$ does not depend on $\mathfrak{E}$ (resp. D). If we start with a different CCT supercategory of $\mathfrak{A}$ we will end up with the same $\mathfrak{B}$ (up to isomorphism). In the following we will exhibit internal conditions which characterize $\mathfrak{B}$.

9. Definition. A category $\mathfrak{B}$ is called a CCT hull of a subcategory $\mathfrak{A}$ provided the following hold:

(a) $\mathfrak{B}$ is a CCT category,

(b) $\mathfrak{A}$ is closed under the formation of finite products in $\mathfrak{B}$,

(c) $\mathfrak{A}$ is dense in $\mathfrak{B}$,

(d) powers of $\mathfrak{A}$-objects are codense in $\mathfrak{B}$.

In order to characterize those categories which have a CCT hull we need the following fact.

10. Proposition. Any dense embedding $\mathfrak{X} \rightarrow \mathfrak{Y}$ preserves initial sources.

Proof. Let $\left(f_{i}: X \rightarrow X_{i}\right)_{I}$ be an initial source in $\mathfrak{X}$, let $Y$ be a $\mathfrak{Y}$-object and let $g: Y \rightarrow X$ be a function such that for each $i$ in $I$ the map $f_{i} \circ g: Y \rightarrow X_{i}$ is a $\mathfrak{Y}$-morphism. To show that $g$ is a $\mathfrak{Y}$-morphism consider a final epi-sink $\left(g_{j}: X_{j} \rightarrow Y\right)_{J}$ in $\mathfrak{Y}$ with all $X_{j}$ in $\mathfrak{X}$. Then all $f_{i} \circ g \circ g_{j}: X_{j} \rightarrow X_{i}$ are $\mathfrak{Y}$ morphisms and hence $\mathfrak{X}$-morphisms. Initiality of $\left(f_{i}\right)_{I}$ implies that all $g \circ g_{j}: X_{j}$ $\rightarrow X$ are $\mathfrak{X}$-morphisms, hence $\mathfrak{Y}$-morphisms. Finality of $\left(g_{j}\right)_{J}$ implies that $g$ is a $\mathfrak{Y}$-morphism.

11. Theorem (Existence of CCT hUlls). For a category $\Re$ with finite products the following are equivalent:

(a) $\Re$ has a CCT hull,

(b) there exists a finite-product preserving embedding of $\Omega$ into some CCT category,

(c) there exists a dense embedding of $\Re$ into some CCT category.

Proof. Propositions 0(a) and 10. 
12. TheOREM (UNIQUENESS of CCT hULls). Any two CCT hulls of $\mathfrak{A}$ are isomorphic.

Proof. Let $\mathfrak{B}$ and $\mathfrak{B}^{\prime}$ be CCT hulls of $\mathfrak{A}$. For objects $A, A^{\prime}, A^{\prime \prime}$ of $\mathfrak{A}$ the canonical equivalences

$$
\mathfrak{B}\left(A, \mathfrak{B}\left[A^{\prime}, A^{\prime \prime}\right]\right) \simeq \mathfrak{B}\left(A \times A^{\prime}, A^{\prime \prime}\right) \simeq \mathfrak{B}^{\prime}\left(A \times A^{\prime}, A^{\prime \prime}\right) \simeq \mathfrak{B}^{\prime}\left(A, \mathfrak{B}^{\prime}\left[A^{\prime}, A^{\prime \prime}\right]\right)
$$

imply that for any function $f: A \rightarrow \mathfrak{A}\left(A^{\prime}, A^{\prime \prime}\right)$ the following holds:

(*) $A \stackrel{f}{\rightarrow} \mathfrak{B}\left[A^{\prime}, A^{\prime \prime}\right]$ is a $\mathfrak{B}$-morphism iff $A \stackrel{f}{\rightarrow} \mathfrak{B}^{\prime}\left[A^{\prime}, A^{\prime \prime}\right]$ is a $\mathfrak{B}^{\prime}$-morphism.

For each $\mathfrak{B}$-object $B$ the sink $S(B)=\left(g_{i}: A_{i} \rightarrow B\right)_{I(B)}$ consisting of all $\mathfrak{B}$ morphisms with domain in $\mathfrak{U}$ and codomain $B$ is a final epi-sink in $\mathscr{B}$ and the source $T(B)=\left(f_{j}: B \rightarrow \mathfrak{B}\left[A_{j}, A_{j}^{\prime}\right]\right)_{J(B)}$ consisting of all $\mathfrak{B}$-morphisms with domain $B$ and codomain a power of $\mathfrak{A}$-objects, is an initial source in $\mathfrak{B}$. Similarly one defines $S^{\prime}\left(B^{\prime}\right)$ and $T^{\prime}\left(B^{\prime}\right)$ for each $\mathfrak{B}^{\prime}$-object $B^{\prime}$. Since $\mathfrak{B}^{\prime}$ is a CCT hull of $\mathfrak{A},(*)$ and 9 (c), (d) imply that for each $B$ in $\mathfrak{B}$ there exists precisely one $B^{\prime}$ in $\mathfrak{B}^{\prime}$ with the same underlying set as $B$ such that the following equivalent statements hold:

(a) $\left(g_{i}: A_{i} \rightarrow B^{\prime}\right)_{I(B)}$ is a final epi-sink in $\mathfrak{B}^{\prime}$,

(b) $\left(g_{i}: A_{i} \rightarrow B^{\prime}\right)_{I(B)}=S^{\prime}\left(B^{\prime}\right)$,

(c) $\left(f_{j}: B^{\prime} \rightarrow \mathfrak{B}^{\prime}\left[A_{j}^{\prime}, A_{j}^{\prime \prime}\right]\right)_{J(B)}$ is an initial source in $\mathfrak{B}$,

(d) $\left(f_{j}: B^{\prime} \rightarrow \mathfrak{B}^{\prime}\left[A_{j}^{\prime}, A_{j}^{\prime \prime}\right]\right)_{J(B)}=T^{\prime}\left(B^{\prime}\right)$,

(e) $\left(g_{i}: A_{i} \rightarrow B^{\prime}\right)_{I(B)}$ and $\left(f_{j}: B^{\prime} \rightarrow \mathfrak{B}^{\prime}\left[A_{j}^{\prime}, A_{j}^{\prime \prime}\right]\right)_{J(B)}$ are in $\mathfrak{B}^{\prime}$.

It follows immediately that there exists a unique functor $H: \mathfrak{B} \rightarrow \mathfrak{B}^{\prime}$ with $H B=B^{\prime}$ for each $\mathfrak{B}$-object $B$. Obviously $H$ is an isomorphism leaving $A$ fixed.

Having established the uniqueness of CCT hulls it seems natural to characterize them by some universal property. For this we need the following result.

13. Proposition. Any dense embedding $\mathfrak{X} \rightarrow \mathfrak{Y}$ between $C C T$ categories preserves powers.

Proof. Proposition 10 implies that the embedding preserves finite products. Let $\left(X, X^{\prime}\right)$ be a pair of $\mathfrak{X}$-objects. To show that the canonical evaluation map $e: X \times \mathfrak{X}\left[X, X^{\prime}\right] \rightarrow X^{\prime}$ has the universal property in $\mathfrak{Y}$, let $Y$ be a $\mathfrak{Y}$-object and $f: X \times Y \rightarrow X^{\prime}$ be a $\mathfrak{Y}$-morphism. If $\left(g_{i}: X_{i} \rightarrow Y\right)_{I}$ is a final epi-sink in $\mathfrak{Y}$ with the $X_{i}$ belonging to $\mathfrak{X}$ then, for each $i$ in $I$, there exists a unique $\mathfrak{X}$-morphism $g_{i}^{*}: X_{i} \rightarrow \mathfrak{X}\left[X, X^{\prime}\right]$ with $e \circ\left(\mathrm{id}_{X} \times g_{i}^{*}\right)=f \circ\left(\mathrm{id}_{X} \times g_{i}\right)$. One easily constructs a unique function $f^{*}: Y \rightarrow \mathfrak{X}\left(X, X^{\prime}\right)$ such that

$$
e \circ\left(\mathrm{id}_{X} \times f^{*}\right)=f \text { and }\left(\mathrm{id} \times f^{*}\right) \circ\left(\mathrm{id} \times g_{i}\right)=\mathrm{id} \times g_{i}^{*}
$$

for all $i$ in $I$. Since all $f^{*} \circ g_{i}=g_{i}^{*}$ are $\mathfrak{Y}$-morphisms, it follows that $f^{*}: Y \rightarrow \mathfrak{X}\left[X, X^{\prime}\right]$ is a $\mathfrak{Y}$-morphism. 
14. TheOREM (UNIVERSAL PROPERTY OF CCT hULls). Let $E: \mathfrak{A} \rightarrow \mathfrak{B}$ be an embedding of $\mathfrak{A}$ into a CCT hull, and let $F: \mathfrak{A} \rightarrow \mathfrak{D}$ be a dense embedding of $\mathfrak{A}$ into some CCT category. Then there exists a unique dense embedding $G: \mathfrak{B} \rightarrow \mathfrak{D}$ with $F=G \circ E$.

Proof. According to Theorem 8 there exists a factorization

$$
\mathfrak{A} \stackrel{F}{\longrightarrow} \mathfrak{D}=\mathfrak{A} \stackrel{F^{\prime}}{\longrightarrow} \mathfrak{D}^{\prime} \stackrel{F^{\prime \prime}}{\longrightarrow} \mathfrak{D}
$$

such that $F^{\prime}$ is an embedding of $\mathfrak{A}$ into a CCT hull $\mathfrak{D}^{\prime}$ and $F^{\prime \prime}$ is a dense embedding of a bireflective subcategory. By Theorem 12 there exists an isomorphism $H: \mathfrak{B} \rightarrow \mathscr{D}^{\prime}$ with $F^{\prime}=H \circ E$. Hence $G=F^{\prime \prime} \circ H$ is the required dense embedding of $\mathfrak{B}$ into $\mathfrak{D}$. Uniqueness follows from the fact that $G$ preserves initial sources (10) and powers (13).

15. ExAMPLES. (a) The CCT hull of Top is the category of epitopological spaces (= espaces d'Antoine). See P. Antoine [1], A. Machado [24], G. Bourdaud [7], [9].

(b) The CCT hull of the category PreTop of pretopological spaces is the category of pseudotopological spaces ( $=L^{*}$ spaces) (see G. Bourdaud [7], [9]).

(c) The CCT hull of the category of completely regular spaces has been shown by G. Bourdaud [7], [8] to be the category of $c$-embedded limit spaces. Hausdorff $c$-embedded spaces have also been studied intensively by $\mathrm{E}$. Binz [5], D. C. Kent et al. [22] and M. Schroder [30]. Hausdorff spaces do not form a topological category, only a closely related kind of category studied by $L$. D. $\mathrm{Nel}$ [27]. Fortunately most of the salient features of CCT categories remain valid.

(d) The CCT hull of the category of finite topological spaces $=$ the coreflective hull of these spaces in Top $=$ the coreflective hull of the Sierpinski space in Top $=$ the category of topological spaces in which all intersections of open sets are open $\simeq$ the category of preordered sets (reflexive, transitive relations).

This is a counterexample to a result of P. Antoine [1] who claimed that any embedding $F$ of $\mathfrak{A}$ into a CCT category $\mathfrak{\Omega}$ which preserves initial structures and powers has a unique extension $F^{*}: \mathfrak{B} \rightarrow \Re$ preserving initial structures and powers. In fact, if $\Re$ is the category of sequential spaces and $\mathfrak{A}$ is the category of finite topological spaces discussed above then the natural embed$\operatorname{ding} F: \mathfrak{A} \rightarrow \mathfrak{\Omega}$ preserves initial sources and powers, but there does not exist any extension $F^{*}: \mathfrak{B} \rightarrow \Re$ of $F$ preserving initial sources. To see this, let $R$ denote the reals with the usual topology and $D$ the reals with the discrete topology, let $\left(f_{i}: D \rightarrow A_{i}\right)_{I}$ be the source consisting of all continuous functions from $D$ into finite spaces and let $\left(g_{j}: R \rightarrow A_{j}\right)_{J}$ be the source consisting of all continuous functions from $R$ into finite spaces. Then both sources are initial in $\Re$. On the other hand the sources $\left(f_{i}: D \rightarrow A_{i}\right)_{I}$ and $\left(g_{j}: D \rightarrow A_{j}\right)_{J}$ are both initial in $\mathfrak{B}$. If there were an extension $F^{*}: \mathfrak{B} \rightarrow \Re$ preserving initial sources, then we would have $D=F^{*} D=R$, a contradiction. The crucial difference 
between Theorem 14 and the claim of Antoine is that in Theorem 14 all embeddings are required to be dense.

16. Remarks. Modifying E. Spanier's [31] construction of quasi-topological spaces, P. Antoine [1], B. Day [13] and O. Wyler [33] demonstrated that categories can be embedded into cartesian closed categories under very mild conditions. However, the resulting supercategories in general fail to have small fibres (hence are not topological in our sense). More seriously, these constructions cannot in general be carried out within a given universe. In fact, it has been shown (H. Herrlich [19]) that there exist nonfull subcategories of Set in which every constant map is a morphism, which cannot be fully embedded into any topological category (even if we allow large fibres) unless we are willing to leave the universe. Thus the problem of finding mild sufficient conditions under which a given $\mathfrak{A}$ will have a CCT hull remains open. Also open is the more specific question: does every topological category have a CCT hull?

By modifying the constructions of the above authors (so as to ensure small fibres) and by using the criterion $O(b)$ the following conditions can be shown to be sufficient for $\mathfrak{A}$ to have a CCT hull (the construction has been outlined in [28]):

(a) $\mathfrak{A}$ has small fibres and every constant map between $\mathfrak{U}$-objects is an $\mathfrak{A}$ morphism,

(b) $\mathfrak{U}$ has finite products and quotients,

(c) in $\mathfrak{A}$, finite products commute with quotients.

Special categories satisfying these conditions include QUnif, Prox, PNear and SNear (quasi-uniform, proximity, pre-nearness and seminearness spaces respectively). Since all topological categories satisfy (a) and (b) it is only (c) that has to be checked. For the cases QUnif and Prox, see Marxen [25].

\section{REFERENCES}

1. P. Antoine, Etude élémentaire des catégories d'ensembles structurés. I, II, Bull. Soc. Math. Belge 18 (1966), 142-164; ibid 18 (1966), 387-414. MR 34 \#220; 35 \#4135.

2. A. Bastiani, Applications différentiables et variétés différentiables de dimension infinite, $\mathrm{J}$. Analyse Math. 13 (1964), 1-114. MR 31 \# 1540.

3. H. L. Bentley, H. Herrlich and W. A. Robertson, Convenient categories for topologists, Comment. Math. Univ. Carolinae 17 (1976), 207-227.

4. E. Binz, Bemerkungen zu limitierten Funktionenalgebren, Math. Ann. 175 (1968), 169-184. MR 36 \#4513.

5. - Continuous convergence on $C(X)$, Lecture Notes in Math., vol. 469, Springer-Verlag, Berlin and New York, 1975.

6. E. Binz and H. H. Keller, Funktionenräume in der Kategorie der Limesraume, Ann. Acad. Sci. Fenn. Sec. AI 383 (1966), 1-21. MR 34 \#6706.

7. G. Bourdaud, Espaces d'Antoine et semi-espaces d'Antoine, Cahiers Topologie Géom. Différentielle 16 (1975), 107-134.

8. - Deux caractérisations des c-espaces, C.R. Acad. Sci. Paris Sér A-B 281 (1975), A313A316.

9. - Some cartesian closed topological categories of convergence spaces, Proc. Internat. Conf. Categorical Topology (Mannheim 1975), Lecture Notes in Math., vol. 540, Springer-Verlag, Berlin and New York, 1976, pp. 93-108. 
10. M. Chartrelle, Construction de catégories auto-dominées, C.R. Acad. Sci. Paris Sér. A-B 274 (1972), A388-A391.

11. G. Choquet, Convergences, Ann. Univ. Grenoble Sect. Sci. Math. Phys. 23 (1948), 57-112. MR 10, 53.

12. C. H. Cook and H. R. Fischer, On equicontinuity and continuous convergence, Math. Ann. 159 (1965), 94-104. MR 31 \#3995.

13. B. Day, A reflection theorem for closed categories, J. Pure Appl. Algebra 2(1972), 1-11. MR 45 \#5187.

14. H. R. Fischer, Limesrdume, Math. Ann. 137 (1959), 269-303. MR 22 \#225.

15. H. Herrlich and G. E. Strecker, Category theory, Allyn and Bacon, Boston, Mass., 1973. MR 50 \#2284.

16. H. Herrlich, Topological functors, Gen. Topology and Appl. 4 (1974), 125-142. MR 49 \#7970.

17. - Cartesian closed topological categories, Math. Coll. Univ. Cape Town 9 (1974), $1-16$.

18. Some topological theorems which fail to be true, Proc. Internat. Conf. Categorical Topology (Mannheim 1975), Lecture Notes in Math, vol. 540, Springer-Verlag, Berlin and New York, 1976, pp. 265-285.

19.

20. M. Katětov, On continuity structures and spaces of mappings, Comment. Math. Univ. Carolinae 6 (1965), 257-278. MR 33 \# 1826.

21. D. C. Kent, Convergence functions and their related topologies, Fund. Math. 54 (1964), 125-133. MR 28 \#4509.

22. D. C. Kent, K. McKennon, G. Richardson and M. Schroder, Continuous convergence in $C(X)$, Pacific J. Math. 52 (1974), 457-465.

25. H. J. Kowalsky, Limesrame und Komplettierung, Math. Nachr. 12 (1954), 301-340. MR $17,390$.

24. A. Machado, Espaces d'Antoine et pseudo-topologies, Cahiers Topologie Géom. Différentielle 14 (1973), 309-327.

25. D. Marxen, Quotients of quasi-uniform spaces (preprint).

26. H. Müller, Über die Vertauschbarkeit von Reflexionen und Coreflexionen Quasi-Top Kategorien über Meng (preprint).

27. L. D. Nel, Initially structured categories and cartesian closedness, Canad. J. Math. 27 (1975), 1361-1377.

28. - Cartesian closed topological categories, Proc. Internat. Conf. Categorical Topology (Mannheim 1975), Lecture Notes in Math., vol. 540, Springer-Verlag, Berlin and New York, 1976, pp. $439-451$.

29. H. Poppe, Compactness in general function spaces, V.E.B. Deutscher Verlag Wiss., Berlin, 1974.

30. M. Schroder, Solid convergence spaces, Bull. Austral. Math. Soc. 8 (1973), 443-459. MR 47 \#9528.

31. E. Spanier, Quasi-topologies, Duke Math. J. 30 (1963), 1-14. MR 26 \# 1847.

32. O. Wyler, Convenient categories for topology, General Topology and Appl. 3 (1973), 225-242. MR 48 \#2972.

33. - Are there topoi in topology? Proc. Internat. Conf. Categorical Topology (Mannheim 1975), Lecture Notes in Math., vol. 540, Springer-Verlag, Berlin and New York, 1976, pp. 699-719.

Department of Mathematics, Universität Bremen, Bremen, Federal Republic of Germany

Department of Mathematics, Carleton University, Ottawa , Ontario, Canada 